\title{
Relativistic outflows from compact objects and generation of Astroparticles
}

\author{
Guy Pelletier* \\ IPAG, CNRS-UJF, Grenoble, France \\ E-mail: Guy.Pelletier@obs.ujf-grenoble.fr
}

Martin Lemoine

IAP, CNRS-UPMC, Paris, France

E-mail: lemoinediap.fr

\section{Illya Plotnikov}

IPAG, CNRS-UJF, Grenoble, France

E-mail: Illya.Plotnikov@obs.ujf-grenoble.fr

\begin{abstract}
High Energy Astrophysics relates to the environment of compact objects that manifest themselves through the generation of non-thermal radiation from radio band up to very high energy gamma rays, cosmic ray generation up to the Ultra High Energy range (a few $10^{20} \mathrm{eV}$ ), very high energy neutrinos (as recorded now by Icecube observatory); in the future, we can expect the detection of Gravitational Waves produced by the formation of compact objects. There are two major physical processes that underline that manifestation, first the formation of relativistic jets, especially in the vicinity of Black Holes, second the formation of relativistic shocks that dissipate their energy with the production of electromagnetic turbulence and astroparticles. These two major processes are paradoxical in the sense that they do not rely on common physical sense and require some inquiry involving non trivial mechanism of General Relativity or non trivial mechanism of collisionless micro-physics. These two processes will be briefly presented with an update of their status.
\end{abstract}

Symposium "Frontiers of Fundamental Physics 14"

15-18 July 2014

Aix Marseille University (AMU) Saint-Charles Campus, Marseille, France

\footnotetext{
*Speaker.
} 


\section{Two physical paradoxes}

Two major processes govern the manifestation of high energy astrophysics. Compact objects, in particular Black Holes, eject plasmas from their environments at relativistic speeds. Then these relativistic outflows collide with the ambient medium, giving rise to shocks producing energetic particles and radiations, revealing the underlying cosmic phenomenon. Relativistic ejection from Black Hole environments and their associated collisionless relativistic shocks are the two major phenomena that are based on physical paradoxes.

A fastly rotating Black Hole, described by the Kerr geometry of space-time, when embedded in a magnetic field, is able to produce relativistic flows along its rotation axis, to the expense of its rotation energy. This is the celebrated Blandford-Znajek mechanism [1]. The paradox of this outflow generation is two-fold. First, a poloidal magnetic field threading the so-called "ergosphere" of the Black Hole gives rise to an electro-motive force due to space-time rotation. Second, a sort of electromagnetic Penrose effect allows to give rise to an energy outflux.

As for the collisionless relativistic shocks, their nature is currently under investigation, and they are able to produce particles of energy much larger than the encountered energy in the centre of mass frame. How is it possible?

Let us examine these two paradoxes successively.

\section{Ejection from a Kerr Black Hole}

The energy outflux results from a mechanism similar to a Penrose one, it is associated with a locally convergent Poynting flux towards the Black Hole and also a convergent flux of negative (with respect to BH rotation) angular momentum. Then there is an outflux of energy and, at far enough distance, the energy flux becomes a Poynting outflux. Let us explain these points a little bit. That kind of description makes sense in a special frame, called ZAMO frame for Zero Angular Momentum Observers. It is built with respect to a more fundamental system of coordinates, the Boyer-Lindquist system of coordinates, that has been derived from the equations of General Relativity by assuming stationarity and axial symmetry and provides the appropriate metric, the Kerr metric, that defines a $d s^{2}$ (see fig.1). The metric involves two scalar functions $(\alpha, \omega)$, the laps function $\alpha(r, \theta)$ is the ratio of a locally measured duration over its corresponding duration measured in universal time, and the function $\omega(r, \theta)$ measures the angular velocity of the system of coordinates. There are two surfaces enveloping the Black Hole, i) the horizon where $\alpha(r, \theta)$ vanishes, and thus where the velocity of light vanishes (maximum gravitational red shift), ii) the ergosphere, containing the horizon (see fig. 1), inside which any physical body, including photons, is enforced to co-rotate with space-time. That rotation is solid on the horizon and defines the Black Hole rotation. The ZAMO frame is defined for each value of the radial coordinate, with a boost velocity $\vec{\beta}$ which is nothing but a sheared rotation of angular velocity $-\omega$.

In the Kerr metric, the Maxwell equations are modified. Recently an elegant and helpful expression of the modification has been derived by S. Komissarov [2], which clearly shows that the presence of a poloidal magnetic field, $\vec{B}_{p}$, threading the ergosphere produces an electro-motive field $\vec{\beta} \times \vec{B}_{p}$ (see fig. 2), as if it were a conductor. This is a first paradox: this is not a material conductor, yet it conducts electric current and drives it, thanks to that property of vacuum described by Kerr's 
metric with a magnetic field. Then in the presence of a tenuous plasma of $e^{+} e^{-}$pairs, likely produced in such an environment, a current is driven that generates a toroidal component of magnetic field, $B_{\phi}$. Actually the black hole environment is enveloped by a "watershed surface": inside the watershed surface matter falls down in the black hole, whereas outside matter is carried away by the Poynting outflux. The magnetic energy is supposed to largely dominates matter energy. Infalling matter carries a total angular momentum, kinetic and magnetic, the dominant magnetic contribution makes it negative: $\ell \propto B_{\phi} \propto I<0$. It can be shown that the flux of angular momentum $\vec{F}_{\ell} \propto-B_{\phi} \vec{B}_{p}$. Whereas in the ZAMO frame, the Poynting flux points towards the black hole horizon, as happens in the environment of a conductor, the total energy flux points outwards because of the influx of negative angular momentum. This is similar to what happens in Penrose's effect (see details in [7], see also [5], [6]); this is an electrodynamics generalization of it, which has been recently put into a more general statement [4]. However one should not forget, as claimed by S. Komissarov [2] and also by R. Blandford himself [3], that the primary aspect of the Blandford-Znajek mechanism is the modification of Maxwell equations by the Kerr metric introducing an electromotive force. Far from the ergosphere, the energy outflux takes the form of a Poynting flux, thanks to a reversal of the electric field on the watershed surface (a ZAMO feature).

The electromotive force is proportional to space rotation $\omega$ which equals $\Omega_{B H}$ on the horizon and a counter electromotive force develops with field line rotation $\Omega_{*}$. The power outflux is maximum when $\Omega_{*}=\Omega_{B H} / 2$, which is presented as a kind of impedence adaptation of the environment with the generator that has a surface resistence on the horizon equals to vacuum resistence. Actually there is also another aspect of that adaptation which is the continuity of the current density on the watershed surface, that plays the role of a kind of critical surface on which the poloidal velocity of the fluid changes its sign.

A few details (see [10], [5], [6], [7]). More precisely the electric field is

$$
\vec{E}=B_{p} \frac{\left(\omega-\Omega_{*}\right) \bar{r}_{e_{t}}}{\alpha c},
$$

( $\bar{r}$ is the circumferencial radius) where one can see that the contribution in $\omega$ is the electromotive force associated to the poloidal field threading the vacuum in the ergosphere, $\Omega_{*}$ describing the rotation of field lines produce a counter electromotive force ( $\bar{r}$ is the cylindrical radius, $\vec{e}_{t}=\vec{e}_{p} \times \vec{e}_{\phi}$ the transverse unitary vector). The toroidal field is generated by the current driven by the electromotive force and reduced by the rotation of field lines:

$$
B_{\phi}=B_{p} \frac{\left(\omega-\Omega_{*}\right) \bar{r}}{\alpha v_{p}}
$$

The poloidal projection of the velocity of matter $v_{p}$ vanishes and changes its sign on the "watershed surface". It is a kind of critical surface where the regularity condition is that $\omega_{\mid w s}=\Omega_{*}$, and thus the electric field vanishes and changes its sign on this surface, whereas the toroidal magnetic field keeps a non vanishing continuous value. After passing the watershed surface, the current $I=\alpha \bar{r} B_{\phi} / 2$ becomes $\propto-\Omega_{*} \bar{r}^{2} /(-c)$, whereas before passing it, the current is $\propto\left(\Omega_{B H}-\Omega_{*}\right) \bar{r}^{2} / c$. Thus for the optimum condition $\Omega_{*}=\Omega_{B H} / 2$, the current passes smoothly the watershed surface, it keeps the same value on a cylinder of circumferencial radius $\bar{r}$. 
The two previous equations lead to the Poynting flux measured by ZAMOs (rescaled by $\alpha$, for providing energy measurement at infinity):

$$
\vec{S}=\alpha^{2} \frac{c}{4 \pi} \vec{E} \times B_{\phi} \vec{e}_{\phi}=\frac{1}{4 \pi v_{p}}\left(\omega-\Omega_{*}\right)^{2} \vec{r}^{2} B_{p} \vec{B}_{p}
$$

As already indicated, the Poynting flux converges towards the horizon inside the watershed surface, whereas it becomes an outflux outside. But there is an inflow of negative angular momentum in the inner region, the flux of angular momentum being obtained from the component $T_{\phi p}$ of the energy-momentum tensor (rescaled by $\alpha$ ):

$$
\vec{F}_{\ell}=-\alpha \frac{c}{4 \pi} \bar{r} B_{\phi} \vec{B}_{p}=-\frac{1}{4 \pi v_{p}}\left(\omega-\Omega_{*}\right) \bar{r}^{2} B_{p} \vec{B}_{p},
$$

so that the total energy flux is always pointing outwards:

$$
\vec{F}_{\varepsilon}=\vec{S}+\omega \vec{F}_{\ell}=-\frac{1}{4 \pi v_{p}} \Omega_{*}\left(\omega-\Omega_{*}\right) \vec{r}^{2} B_{p} \vec{B}_{p}
$$

This is similar to a Penrose process that is based on the fact that the energy invariant $\varepsilon_{\infty}$ (or energy at infinity) depends on both the local energy measurement $\varepsilon$ and the local angular momentum measurement $\ell$, such that $\varepsilon_{\infty}=\alpha \varepsilon+\omega \ell$. Thus in the ergosphere, a body of negative angular momentum can have a negative energy. Suppose a falling body of positive energy $\varepsilon_{0}$ that splits in the ergosphere into a piece having a positive angular momentum and energy $\varepsilon_{1}$ and another one having a negative angular momentum and a negative energy $\varepsilon_{2}$. The body of positive energy $\varepsilon_{1}$ moves along an outwards geodesic, whereas the body of negative energy $\varepsilon_{2}$ falls towards the horizon. Since the energy invariant is additive, the outwards body got an energy $\varepsilon_{1}$ larger than the initial energy $\varepsilon_{0}$ at the expense of Black Hole rotation, because $\varepsilon_{1}=\varepsilon_{0}-\varepsilon_{2}>\varepsilon_{0}$. This is exactly what happens with the electromagnetic fluxes of energy and momentum in the Blandford-Znajek mechanism.

For $\Omega_{*}=\Omega_{B H} / 2$, the power is maximum and one obtains the same value at the Horizon and at infinity:

$$
\vec{F}_{\varepsilon}=\frac{\Omega_{*}^{2} \vec{r}^{2}}{4 \pi c} B_{p} \vec{B}_{p}
$$

which leads to the maximum power, and for a given spin "a" of the Black Hole, the maximum power reaches the considerable following value, which is comparable with the accretion power:

$$
P_{B Z} \simeq a^{2}\left(\frac{B_{n}}{10^{4} G}\right)^{2}\left(\frac{M_{*}}{10^{8} M_{\odot}}\right)^{2} 10^{44} \mathrm{erg} / \mathrm{s} .
$$

That high value suggests that the process can explain the powering of the extragalactic jets.

Impressive numerical simulations have been performed recently [8] that show the possibility to get an ejection power larger than the accretion power and proportional to the square of the spin "a". However, despite its strong interest, this process is not the only candidate for the formation of relativistic jets. Indeed accretion disks thread by opened magnetic field lines are also capable to produce jets with a comparable power even larger than the accretion power [11]. 
- Spinning Black Hole with an accretion disk carrying a magnetic field

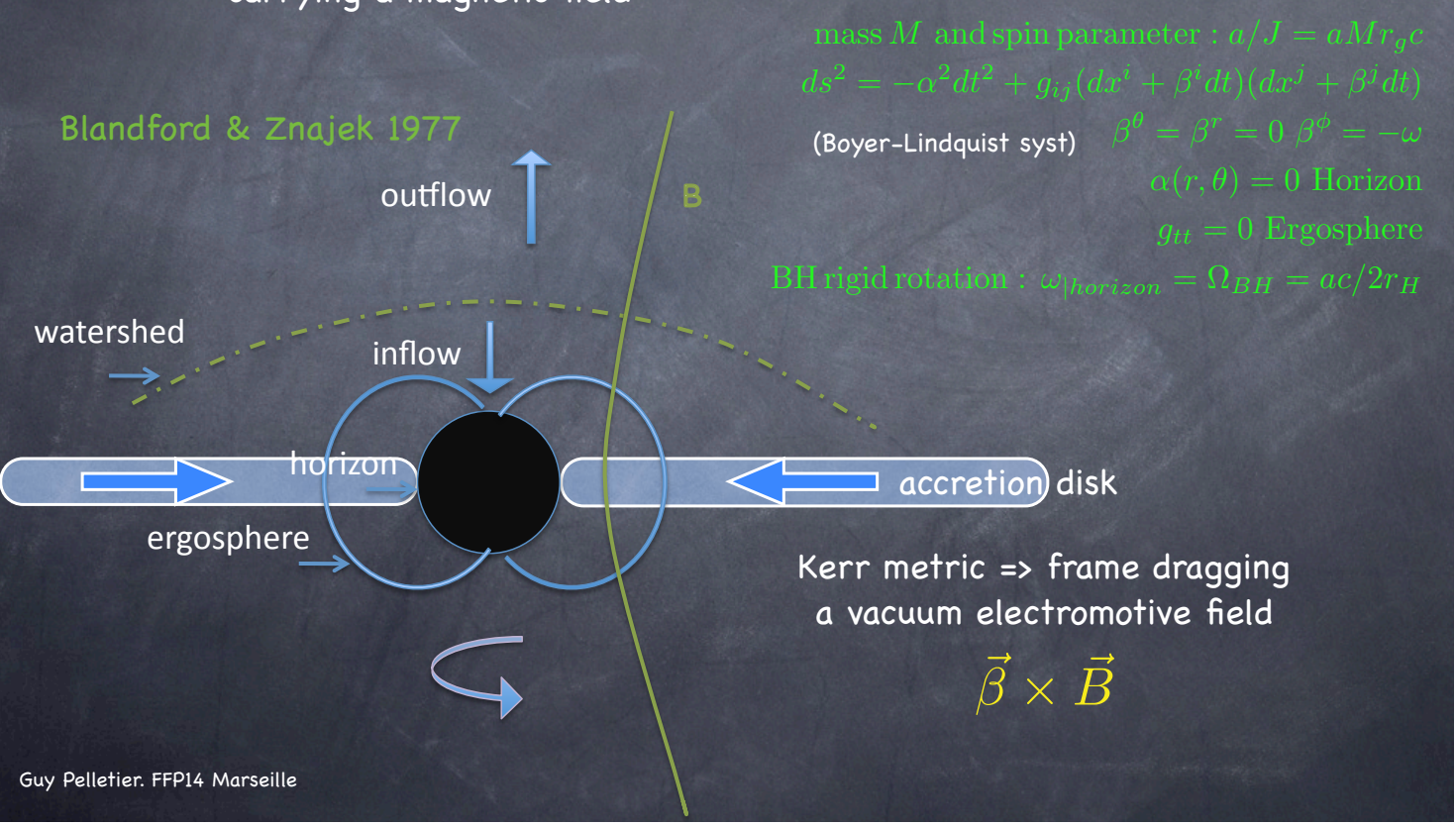

Figure 1: Spinning Black Hole thread by a magnetic field carried by an accretion disk.

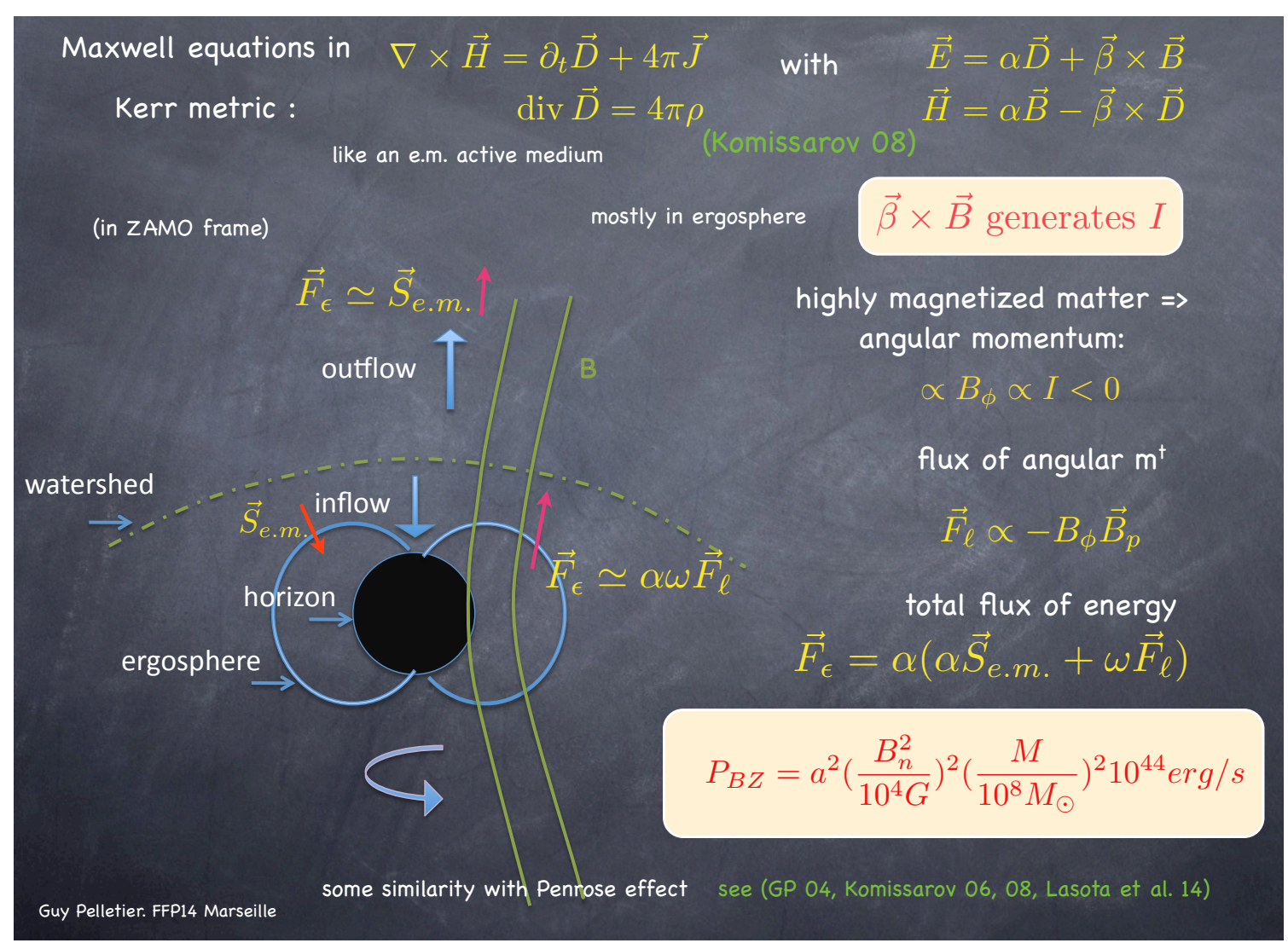

Figure 2: Angular momentum and energy budget, like a Penrose mechanism. 


\section{What is a collisionless relativistic shock?}

Relativistic shocks involve high energy particles whose mean free path is much larger than the size of the shock region. Hence a collisionless front has to be designed. If, for any reason, some electromagnetic energy rises somewhere in a plasma flow, with an intensity high enough with respect to the kinetic energy of the flow for reflecting a sizable fraction of incoming particles, then we can expect that some nonlinear front is set up. Indeed the back stream of returning particles can generate the expected electromagnetic turbulence through an appropriate interaction of the returning stream with the incoming stream. The idea of a collisionless shock is such a self-sustaining nonlinear front developing at micro-scales. Downstream, according to ultra-relativistic kinematics, the distribution function is supposed to isotropize in a frame moving at $c / 3$ with respect to the front. Particles undergo some thermalization with a temperature $T \simeq 0.2 \gamma_{s} n m_{p} c^{2}$, where $\gamma_{s}$ is the Lorentz factor of the shock front with respect to the background plasma. This supposes a collisionless dissipation which cannot be else than Landau damping (linear or nonlinear, with Tcherenkov or synchrotron resonances) and because this is governed by the Vlasov equation which describes an isentropic evolution, the whole information is preserved unless phase space ripples are smoothed out (C. Villani [12]).

The interaction between returning and incoming streams at relativistic speed triggers various micro-instabilities; however one of them plays a major role, because it survives background electron preheating and beam dispersion better than the others, namely the Weibel instability. The Weibel instability generates mostly magnetic micro-turbulence, as will be presented further on, which produces particle scattering at a high rate that ensures the isotropization of the downstream distribution and also the formation of a high energy tail of supra thermal particles, thanks to a Fermi process. Now, despite its magnetic nature, an efficient heating is expected for both protons and electrons.

From theory and simulations we expect to know a few parameters that are essential for High Energy Astrophysics, especially the factors $\xi_{c r}, \xi_{B}$ of conversion of incoming energy density into cosmic rays (or more precisely into supra thermal particles) and into magnetic turbulence:

$$
\begin{aligned}
P_{c r} & =\xi_{c r} \gamma_{s}^{2} n m c^{2} \\
\frac{B^{2}}{4 \pi} & =\xi_{B} \gamma_{s}^{2} n m c^{2},
\end{aligned}
$$

where $P_{c r}$ is the pressure of supra thermal particles at the shock front; and the parameter $\xi_{B}$ is measured at the shock front as well.

This scenario of collisionless relativistic shocks has been plainly confirmed in remarkable numerical simulations published in 2008 by A. Spitkovsky [13]. The first simulations have been performed for $e^{+} e^{-}$-plasma without a mean field and then extended to electron-ion plasma including a mean field [14]. These were historical papers that provoked a new impetus in the physics of high energy phenomena in astrophysics.

\section{Weibel turbulence}

\subsection{Excitation}

The stream of returning particles (reflected and/or backscattered from downstream when Fermi 
process is operative) penetrate in the upstream (background) plasma in the form of a very collimated ultra-relativistic beam that triggers micro-instabilities in the background plasma. Weibel instability is favored, especially in a very weakly magnetized plasma [17], and it works as follows. Suppose that a magnetic wave has been excited in a transverse direction with respect to the beam. The associated Lorentz force separates opposite charges of the beam, that was initially neutral. Because they are traveling at relativistic speed with respect to the background plasma, an electric current is generated which amplifies the original magnetic wave; thus it blows up as described by the following system of equations. Maxwell-Ampère equation for the potential vector along the beam $A$ contains a supplementary current due to charge separation $\delta \rho$ :

$$
\left(\partial_{t}^{2}-c^{2} \Delta+\omega_{p}^{2}\right) A=4 \pi c^{2} \beta_{b} \delta \rho,
$$

- in this short presentation, $\omega_{p}$ is the plasma proper frequency of the background plasma, that can be composed of electrons and positrons, like in the pulsar wind; in that case the plasma frequency, $\omega_{p}^{2} \equiv 4 \pi n_{0} e^{2} / m$, contains the electron mass. In a proton electron plasma, when the electrons are hot enough to be considered in a local Boltzmann equilibrium, the dynamics is that of protons and the plasma frequency contains the proton mass - and the charge separation due to the Lorentz force evolves so that

$$
\partial_{t}^{2} \delta \rho=-\frac{\omega_{p}^{2}}{4 \pi} \frac{\xi \beta_{b}}{1+\xi} \Delta A
$$

where $\xi \equiv \omega_{p b}^{2} / \omega_{p}^{2} \simeq \xi_{c r}\left(\omega_{p b}\right.$ being the beam plasma frequency). The growth rate is then

$$
g(k)=\xi^{1 / 2} \frac{k v_{0}}{\left(1+k^{2} \delta^{2}\right)^{1 / 2}},
$$

which reaches a maximum value $\sqrt{\xi} \omega_{p}$ at a scale $\delta \equiv c / \omega_{p}$. The instability is fast since it grows over a few plasma period. This is the electron plasma characteristics in an pair plasma, the proton characteristics in a proton-electron plasma (assuming warm electrons in quasi Boltzmann equilibrium).

\subsection{Proper frame and scattering, front frame and heating}

The statement of a "magnetic turbulence" is not co-variant, this is true in a well-defined frame, that can be found ([19], [18]) to move at a velocity $\beta_{m}$ such that

$$
\beta_{m}=\frac{\omega_{p}^{2} \beta_{p}+\omega_{b}^{2} \beta_{b}}{\omega_{p}^{2}+\omega_{b}^{2}} .
$$

In the background frame, it moves forwards at a non-relativistic speed $\beta_{W \mid b g}=\xi / 1+\xi$; in the front frame it moves backwards at ultra relativistic speed $\beta_{W \mid f}=-\beta_{s}(1-\xi) /(1+\xi)$, a little less faster than the upstream flow. In that Weibel frame, the instability is purely growing, purely magnetic without any Poynting flux; and particles undergo scattering without any variation of their energy. In the front frame, the magnetic field (transverse) is amplified by $\gamma_{s}$, there is an electrostatic field (in the transverse direction) of same intensity, a magnetic pressure $P_{W}=\left(\xi_{B} / 2\right) \gamma_{S}^{2} n_{0} m c^{2}$ and a strong Poynting flux towards the shock front, $S_{W}=-\xi_{B} \gamma_{s}^{2} n_{0} m c^{2}$; and particles undergo both scattering and heating. The conversion parameter $\xi_{B} \sim \xi_{c r}$ according to conservation laws [17], with 
$\xi_{c r} \sim 10 \%$ according to numerical simulations. The electrons are strongly preheated in the shock precursor because of the strong electrostatic field and the intense scattering. Protons experience also a similar, but moderate, preheating. The particles reach an effective transverse temperature $T \simeq e \Phi_{r m s}$ where $\Phi_{r m s}$ is the rms value of the electrostatic random potential (see [19]) produced in the front frame and $e \Phi_{r m s} \simeq \gamma_{s} e B_{r m s} \bar{\delta}_{e}$; since $e B_{r m s} \delta_{i}=\xi_{B}^{1 / 2} m_{p} c^{2}$ and the inertial length of the relativistically hot electrons $\bar{\delta}_{e}=\xi_{B}^{1 / 2} \delta_{i}$ we obtain an estimate of the effective transverse temperature achieved close to the shock front:

$$
T \simeq \xi_{B} \gamma_{s} m_{p} c^{2}
$$

The heating process occurring in the precursor is almost sufficient for the formation of the shock transition. That kind of effective heating of protons can also be seen as resulting from trapping of their transverse momentum in the quiver force. However the Weibel turbulence is made of a continuum of modes that does not allow to treat the instability saturation as a simple trapping mechanism, but the estimate of the saturation level is almost the same. The description of particles dynamics in the intense magnetic and electrostatic fields in the front frame [19] seems more straightforward.

Because of that temperature, the thermal pressure force is able to balance the Lorentz force in the process of charge separation responsible for Weibel instability, which thus saturates in the form of filaments resulting from the transverse Boltzmann equilibrium between the two forces. Like in 2D hydrodynamical turbulence, where pairing of vortices of opposite polarity takes place, Weibel turbulence gives rise to pairing of filaments of opposite currents. This is described by a sinh-Poisson equation:

$$
\Delta A=-\sinh A
$$

where $A$ stands for $\beta_{b} e A / T$ and the scale unit is $\bar{\delta}_{e}$.

The particle scattering rate is properly estimated in the Weibel frame and turns out to be inversely proportional to the square of particle energy (numerically checked in [19]):

$$
v_{s} \simeq \frac{e^{2} B^{2} \delta_{i}}{m^{2} c^{3} \gamma^{2}}
$$

For protons in the Weibel or background frame, $v_{s} \sim \xi_{B} \omega_{p i} / \gamma^{2}$. That decrease of scattering in $\gamma^{-2}$ is the weak point of Fermi acceleration at relativistic shocks that limits their efficiency in the detailed balance.

\subsection{Fermi process and its inhibition at relativistic shocks}

It has been argued both theoretically and numerically [20], [15], that the Fermi acceleration of particles, based on particle scattering on both sides of a shock, is inhibited by a mean field at relativistic shocks. The reasons are, i) that the transverse component of the mean field is seen amplified by the shock Lorentz factor at the shock front, thus the field is almost perpendicular, ii) particles returning from downstream to upstream penetrate in the precursor with a pitch angle smaller than $1 / \gamma_{s}$, iii) because of Larmor deflection, they are caught up by the shock in a time much smaller than Larmor time. Fermi process is thus possible only if scattering occurs on a time scale shorter than the Larmor time in the background magnetic field.

In the downstream flow, a particle is entrained by the "frozen in" magnetic field unless efficiently scattered in a time shorter than its local Larmor time (in the background magnetic field) 
[15], $v_{s}(\gamma)>\omega_{L}(\gamma)$. This leads to a necessary condition on the turbulence level: $\sigma<\xi_{B}^{2}$ and to a maximum particle energy:

$$
\gamma<\gamma_{s} \frac{\xi_{B}}{\sqrt{\sigma}} .
$$

In the upstream flow, it is required that a penetrating particle be scattered on a time scale shorter than the Larmor deflection time for an angle $1 / \gamma_{s}$. One obtains the same above criterium, expressed in the upstream rest-frame. This is a severe constraint, even at low magnetization. For instance, in the case of the termination shock of a Gamma Rays Burst in a medium having the characteristic parameters of the interstellar medium of our galaxy, the magnetization is $\sigma \sim 10^{-9}$. With $\xi_{B}=$ $0.1-0.01$, there is room for developing a Fermi process at a relativistic shock having $\gamma_{s}=300$ typically. However protons are accelerated up to an energy $\sim 10^{16} \mathrm{eV}$. This is not bad, but it cannot explain the generation of Ultra High Energy Cosmic Rays (a few $10^{20} \mathrm{eV}$ ).

Now there is also a threshold condition for the excitation of micro-turbulence; indeed the precursor length controlled by the mean field has to be large enough for the growth of instability. We have shown [18] that Weibel instability grows when $\sigma<\xi_{c r}^{3}$. Actually numerical simulations [21] indicates that micro-turbulence is excited for $\sigma$ larger than $\xi_{c r}^{3}$. We found [18] that this is due to a peculiar filamentation instability, similar to Weibel instability, triggered by the net current generated by the charge separation produced by the mean field. The threshold condition is then increased to $\sigma<\xi_{c r}^{2}$. That condition is fairly close to the condition for scattering $\sigma<\xi_{B}^{2}$, since $\xi_{B} \sim \xi_{c r} \sim 0.1$. It is quite remarkable that these thresholds are independent on the shock Lorentz factor (there is a kind of buffer effect explained in [18]). Roughly, we can say, in agreement with numerical simulations that the transition towards Fermi process is $\sigma<10^{-3}$.

\section{Radiation from relativistic shocks; Gamma Ray Bursts}

As for the acceleration of electrons, the main limitation is not due to the inhibition of scattering by the mean field, but by radiation cooling. They radiate a synchrotron-like emission while being scattered off magnetic turbulence. In both upstream and downstream frames, they have the same maximum energy (measured locally), independent of the magnetic field intensity (in agreement with [22]):

$$
\gamma_{\max }=\left(\frac{\delta_{i}}{r_{e}}\right)^{1 / 3}
$$

where $r_{e} \equiv e^{2} / m_{e} c^{2}$ is the electron classical radius. This is an almost universal limit because its dependence with local parameters is only in $n^{-1 / 6}$, and put the limit Lorentz factor between $10^{6}-10^{7}$. However the corresponding energy of the emitted photons is very sensitive to the shock Lorentz factor and to the nature of the incoming medium. Indeed, in the case of Gamma Ray Burst, the fact that the incoming plasma is made of protons and electrons, allows to reach a very high photon energy (measured by the observer) because of the generation of a very intense magnetic field:

$$
\varepsilon_{p h}=\gamma_{s}^{2} \xi_{B}^{1 / 2} \frac{m_{p} c^{2}}{\alpha_{f}}\left(\frac{r_{e}}{\delta_{i}}\right)^{1 / 3} \sim 10 \mathrm{GeV} \text { for } \gamma_{s}=300
$$

where $\alpha_{f}$ is the fine structure constant. 
Such high energy emission from early afterglows was observed, and possible emission beyond $10 \mathrm{GeV}$ has been interpreted as a synchrotron self-Compton effect [23]. The generation (not amplification of a pre-existing field) of such intense magnetic field is an important result and, because diagnosed by its synchrotron emission, its evolution can be followed in the GRB afterglow [23].

\section{The UHECR issue and physics of mildly relativistic shocks}

Looking at Fermi acceleration at a shock, one can see that the performances increase with the velocity of the shock. However we have seen that ultra-relativistic shocks, that are entirely described by micro-physics, can produce the most intense radiation events of the Universe, but not the ultra high energy cosmic rays. This is because the interaction length or time between particles and fields is too short in the precursor with the presence of an avoidable perpendicular mean field. There is more room for interaction when the shock is only mildly relativistic, where the magnetization effect is less stringent. The excitation of MHD turbulence can be expected under some frequent conditions. The physics is more complex; however numerical simulations are easier and laser experiments can be envisaged. The UHE range for cosmic rays can be expected from those mildly relativistic shocks if they occur in an ultra relativistic flow pointing towards the observer, like in AGN jets or GRB outflows.

\section{Acknowledgments}

This work has been supported by the C.N.R.S. Programme National Hautes Énergies (PNHE).

\section{References}

[1] R. Blandford, R. Znajek 1977, MNRAS, 179, 433.

[2] S. Komissarov 2009, JKPS, 54, 2503, and private communication.

[3] R. Blandford 2010, private communication.

[4] J.P. Lasota, E. Gourgoulhon, M. Abramowicz, A. Tchekhovskoy, R. Narayan, 2014, Phys. Rev. D, 89, 024041.

[5] V. Beskin, I. Kuznetsova, 2000, NCimB.115..795B.

[6] M. Camenzind, 1989, Accretion Disks and Magnetic Fields in Astrophysics. Kluwer, Dordrecht.

[7] G. Pelletier 2003, arXiv:astro-ph/0405113, "Black Hole Induced Ejections", in "Dynamics and dissipation in electromagnetically dominated media" (Nova Science) edited by M. Lyutikov.

[8] A. Tchekhovskoy, J. McKinney, R. Narayan, 2012, MNRAS, 372, 2040.

[9] J. McKinney, A. Tchekhovskoy, R. Blandford, 2012, MNRAS, 423, 3083.

[10] K. Thorne, R. Price, D. MacDonald, 1986, “Membrane Paradigm”, Yale University Press.

[11] G. Ghisellini, F. Tavecchio, L. Maraschi, A. Celotti, T. Sbarrato, 2014, Nature, 515, 376.

[12] C. Villani, 2014, Phys. of Plasma, 21, 030901. 
[13] A. Spitkovsky, 2008, Astrophys. J., 682, L5.

[14] L. Sironi, A. Spitkovsky, 2011, Astrophys. J., 726, 75.

[15] G. Pelletier, M. Lemoine, A. Marcowith, 2009, MNRAS, 393, 587.

[16] M. Lemoine, G. Pelletier, B. Revenu, 2006, ApJL, 645, L129.

[17] M. Lemoine, G. Pelletier, 2010, MNRAS, 402, 321; 2011, MNRAS, 417, 1148.

[18] M. Lemoine, G. Pelletier, L. Gremillet, I. Plotnikov, 2014, MNRAS, 440, 1365; EPL, 106, 55001.

[19] I. Plotnikov, G. Pelletier, M. Lemoine, 2013, MNRAS, 430, 1280.

[20] J. Niemiec, M. Ostrowski, M. Pohl, 2006, Astrophys. J., 650, 1020.

[21] L. Sironi, A. Spitkovsky, J. Arons, 2013, Astrophys. J., 771, 54.

[22] J. Kirk, B. Reville, 2010, Astrophys. J., 710, 16.

[23] M. Lemoine, 2013, MNRAS, 428, 845; M. Lemoine, Z. Li, X.Y. Wang, 2013, MNRAS 435, 3009. 